\title{
Culminación de estudios: Una propuesta integradora en la Educación Superior
}

\section{Completion of studies: An integrative proposal in Higher Education}

\author{
Lorna Cruz Rizo ${ }^{1}$ \\ Lorna.cruzrizzo@yahoo.com \\ Eneida Catalina Matos Hernández ${ }^{2}$ \\ Recibido: 1/09/2016, Aceptado: 1/11/2016
}

\begin{abstract}
RESUMEN
Dadas las dificultades en el proceso de culminación de estudios en Educación Superior, los estudios teóricos y prácticos desarrollados resultan sorprendentemente escasos. Históricamente, ha existido una limitada cantidad de estudiantes que sustentan sus tesis de grado después de completar créditos en la Escuela de Lenguas y Lingüística de la Universidad de Guayaquil. Consecuentemente, el problema de esta investigación es: insuficiencias en el proceso de culminación de estudios en relación con la gestión de la formación de los estudiantes. Por tanto, devino objetivo el perfeccionar la culminación de estudios para la obtención del título universitario, a través de la implementación de una propuesta praxiológica de integración entre la formación académica y la investigación formativa. La sistematización de experiencias de las autoras dinamizó la metodología utilizada. La modelación de la gestión curricular de la integración entre la formación académica y la investigación formativa se reveló como el aporte principal y solución al problema detectado. Como resultados obtenidos, los estudiantes solucionaron problemas educativos diagnosticados, a la vez que diseñaron la primera versión de sus tesis. Se logró así perfeccionar cualitativa y cuantitativamente este proceso. Esta propuesta puede ser generalizable a cualquier proceso de culminación de estudios a través de tesis de grado.
\end{abstract}

Palabras clave: Culminación de estudios, Educación Superior, formación académica, investigación formativa, tesis de grado

\begin{abstract}
Given the up- dated difficulties in the completion of studies process in Higher Education, theoretical and practical studies developed are surprisingly scarce. Historically, there has been a limited quantity of students that support their degree thesis after completing their credits at the School of Languages and Linguistics at the University of Guayaquil. Consequently, the problem faced by this paper is: insufficiencies in the completion of studies process in relation to the management of the students' formation. Thus, the objective is to improve the completion of studies process for obtaining the university degree, through the implementation of a praxiological proposal of academic formation and formative research integration management. The author's experiences systematization invigorated the methodology used.
\end{abstract}

\begin{tabular}{ll}
\hline 1 & Docente de la Universidad de Guayaquil. Ecuador \\
2 & Docente de la Universidad de Guayaquil. Ecuador
\end{tabular} 


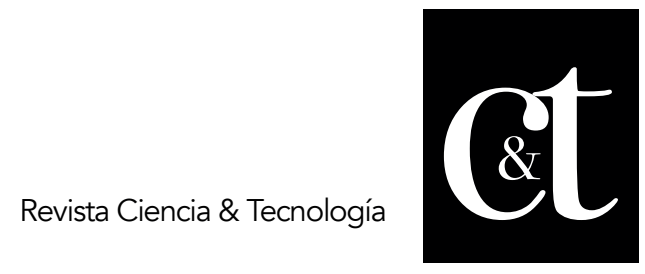

No. 13, 31 de enero de 2017

ISSN impreso: 1390 - 6321

\begin{abstract}
Modeling the curricular management of the academic formation and formative research integration was revealed as the main proposal and solution to the problem diagnosed. As main results obtained, the students were able to solve educative problems diagnosed, while they drew up the first draft of their theses. In this way this process was qualitatively and quantitatively improved. This proposal may be generalizable to any process of completion of studies through degree thesis.
\end{abstract}

Keywords: Completion of studies, Higher Education, academic formation, formative research, degree thesis

\title{
Introducción
}

Dadas las dificultades actuales en el proceso de culminación de estudios a nivel internacional en Instituciones de Educación Superior (IES), los estudios teóricoprácticos realizados al respecto son sorprendentemente exiguos y marcan una brecha a transitar en la Pedagogía de la Educación Superior.

La presente investigación, por tanto, sienta las pautas para posteriores profundizaciones en la diversidad de carreras universitarias. Por su alto nivel de esencialidad, su propuesta de gestión académico- investigativa es potencialmente generalizable a cualquier proceso de culminación de estudios que pretenda entrenar a los estudiantes para enfrentarse con éxito a este proceso e insertarse competentemente en el mundo laboral.

Desde esta perspectiva, el proceso de formación de los profesionales a escala internacional está llamado a perfeccionarse en función de las demandas sociales. La universidad no puede seguir graduando sujetos que no son capaces de responder a los problemas cada vez más complejos y emergentes de sus contextos de actuación.

Interminable sería la lista de investigaciones realizadas en este tema, desde sus diversas perspectivas: gestión, diseño curricular, dinámica de los procesos universitarios, metodología de la enseñanza universitaria, evaluación de procesos de aprendizaje, vínculo con la comunidad, prácticas profesionales, didácticas particulares de cada carrera, entre otras.

Sin embargo, existe un proceso que ha quedado marginado de los numerosos estudios científicos realizados. Teniendo en cuenta una búsqueda exhaustiva de información, realizada por las autoras de este artículo, es posible afirmar que hasta el momento el proceso de culminación de estudios en la educación superior, no ha contado con profundizaciones teóricas o praxiológicas suficientes. Se asume aquí como proceso de culminación de estudios, el estadio final de la formación en un nivel educativo particular, en este caso en la Educación Superior, en el que el estudiante, deberá demostrar por medio de diferentes vías, que además de haber cumplido los créditos establecidos para su carrera, es capaz de un desempeño profesional competente, que le permite integrarse dignamente a la comunidad profesional. Este proceso, por ende, implica la evaluación formativa y sumativa de las competencias profesionales desarrolladas, de modo que sea posible otorgar o no el título de tercer nivel al aspirante.

Por tanto, el presente estudio pretende perfeccionar el proceso de culminación de estudios para la obtención del título universitario, para lo cual muestra, cómo fue aplicada su propuesta praxiológica de integración entre la formación académica y la investigación formativa, mediante la sistematización de experiencias de las autoras 


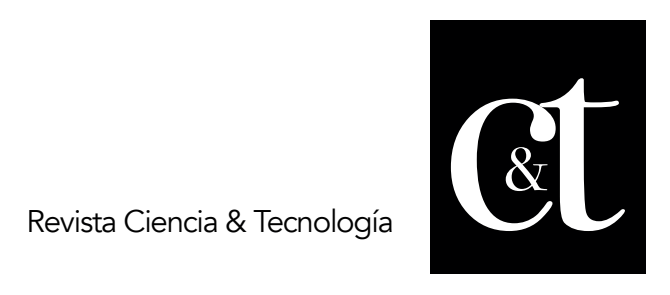

No. 13, 31 de enero de 2017

ISSN impreso: 1390 - 6321

como profesoras titulares de la Universidad de Guayaquil, Ecuador.

De este modo, las autoras logran modelar sistémicamente la gestión curricular de la integración entre la formación académica y la investigación formativa como el aporte principal y solución al problema detectado.

\section{Desarrollo \\ Planteamiento del problema}

Históricamente, en la Facultad de Filosofía, Letras y Ciencias de la Educación de la Universidad de Guayaquil, particularmente en la Escuela de Lenguas y Lingüística han existido dificultades en cuanto al proceso de culminación de estudios, lo que actualmente se conoce, desde los términos legales, como titulación.

Los estudiantes logran vencer los créditos exigidos en la carrera, sin embargo, pasan años sin que logren graduarse por falta del ejercicio de la sustentación de tesis de grado. Dadas estas dificultades, en ocasiones se han tomado medidas de solución operativa y emergente de forma improvisada que, aunque en algún momento han logrado elevar el número de graduados, con vistas a procesos de acreditación de la calidad, no han logrado resolver estas dificultades de manera sostenida.

Una de las causas de esta situación es que dichas medidas no responden a un estudio causal de las verdaderas dificultades que, desde la perspectiva de esta investigación, han estado centradas en el proceso de formación académico e investigativo y han estado frenando el desarrollo de la competencia investigativa de los estudiantes y por tanto, no les permite concretar un proceso de investigación como tesis de grado.

Dada la situación anterior, las autoras de este estudio, realizaron una sistematización bibliográfica, como resultado de la cual -sorprendentemente- se afirma aquí la exigua existencia de estudios teóricos acerca del proceso de culminación de estudios en la Educación Superior. No obstante, es pertinente reconocer los estudios realizados por Jiménez Padilla, Díaz Rodríguez \& Catalá Concepción (2011) respecto a la aplicación de acciones dirigidas a la organización, desarrollo y evaluación del proceso de preparación de los estudiantes de una carrera universitaria para los ejercicios de culminación de estudio, consiguiendo articular al profesor, al tutor y al guía. Sin embargo, desde la posición de esta investigación se considera necesaria una propuesta de mayor nivel de esencialidad científica que pueda ser funcional en una diversidad de carreras, al menos de la misma naturaleza, y donde los roles del docente puedan estar integrados en un solo sujeto, evitándose así la digresión de acciones formativas.

De manera general, por ende, se reconoce que los antecedentes históricos que desde la ciencia sustentan el presente estudio son difusos, ya que a partir de los resultados de dicha búsqueda teórica, es posible aseverar que el proceso de culminación de estudios en las IES (referido a la formación de grado, entiéndase formación universitaria, de tercer nivel) o de titulación, como también es viable llamarle, ha sido mayormente objeto de análisis legal, a nivel internacional y nacional, con el objetivo, de poder precisar las normativas, formas, requisitos y procedimientos en que un estudiante universitario podrá obtener el título que lo acredite como profesional. Sin embargo, en la bibliografía consultada no se encuentran estudios suficientes referidos a teorías, modelos teóricos, principios, a comprender y explicar las esencialidades del proceso de culminación de estudios. 


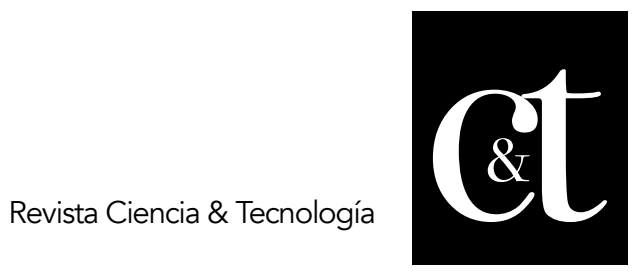

No. 13, 31 de enero de 2017

ISSN impreso: 1390 - 6321

Siendo así, la investigación que aquí se argumenta contribuye a solucionar el problema investigativo dado en las insuficiencias en el proceso de culminación de estudios en relación con la gestión de la formación de los estudiantes.

Este problema de investigación deviene problema científico al ser expresión de las falencias en el proceso de implementación curricular de la culminación de estudios en relación con las insuficiencias teórico- metodológicas, que desde la Pedagogía de la Educación Superior, no logran explicar suficientemente este proceso, lo que limita la respuesta universitaria a las demandas sociales de profesionales graduados.

Lo anterior, entonces, como problema científico de investigación se mueve en un espacio dinámico entre lo práctico y lo teórico, por lo que teniendo en cuenta la inminencia de la necesidad de una respuesta de ciencia, se enfocó desde la perspectiva de una investigación praxiológica, que a la vez de ofrecer soluciones científicas concretas, fuera generando nuevos constructos de ciencia con un nivel de esencialidad suficiente, como para revelar sus regularidades y poder ser implementada en contextos similares.

\section{Antecedentes y fundamentación teórica}

A pesar de la inexistencia de antecedentes teóricos referidos específicamente al proceso de culminación de estudios propiamente dicho, es importante reconocerlo como cúspide de concreción de los procesos de investigación formativa que deben irse generando de manera transversal durante toda la carrera, por ende, es oportuno reconocer que:

Una (...) acepción del término Investigación Formativa es la de "formar" en y para la investigación a través de actividades que no hacen parte necesariamente de un proyecto concreto de investigación. Su intención es familiarizar con la investigación, con su naturaleza como búsqueda, con sus fases y funcionamiento. (Restrepo, 2016 p.8).

La investigación formativa, entonces, dista de la investigación propiamente dicha, en el sentido en que la primera no aspira a lograr grandes aportes científicos como resultado de la investigación, sino que su objetivo fundamental es formar al estudiante en el rigor y la disciplina de los procesos de investigación científica, de modo que pueda él, una vez graduado, ser el artífice de sus propios procesos investigativos, como parte de equipos profesionales. De este modo, el proceso de culminación de estudios, centrado en la elaboración y sustentación de una tesis de grado, no aspira a que el estudiante proponga transformaciones, ni innovaciones trascendentes a la ciencia que estudia, sino que demuestre que es capaz de aplicar el método científico con la ética necesaria.

En tal sentido, el síndrome de Todo Menos Tesis (TMT) o All But Dissertation (ABD), como tendencia mundial que afecta la culminación de estudios en educación superior, (Lani, 2010) también ha estado afectando este contexto de formación de grado; aunque este proceso ha sido diagnosticado en espacios de formación doctoral; al ser considerado como un "viaje" impredecible e incierto, lleno de incertidumbres (Mujtaba, 2007).

Desde la psicología, los estudios realizados por McDermott (2002), Bridgmon (2007) y Blum (2010) profundizan en los conflictos que desde las relaciones intra- e inter psicológicas de los individuos y entre estos y sus contextos socio-familiares de desempeño han estado influyendo en las dificultades para terminar sus tesis de 


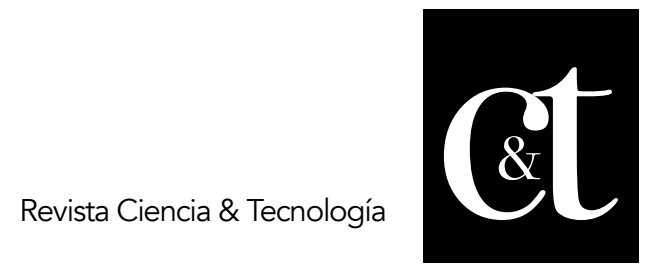

No. 13, 31 de enero de 2017

ISSN impreso: 1390 - 6321

doctorado. Estos estudios, por ende, previa contextualización, devienen un fundamento importante para encaminar los procesos de acompañamiento personalizado de tutoría de tesis, que deben atravesar toda la culminación de estudios, generando espacios de comunicación funcional e integral.

La investigación formativa, entonces, desde la formación de grado, está llamada a lograr que el estudiante logre titularse: "Enseñando a investigar a docentes y estudiantes; desarrollando habilidades cognoscitivas como la analítica, el pensamiento productivo y la solución de problemas; familiarizando a los estudiantes con las etapas de la investigación y los problemas que éstas plantean." (Restrepo, 2016 , p.10). Por tanto, este proceso de culminación de estudios es, en esencia investigación formativa ya que está orientado a la formación académica y profesional dentro de un currículo definido (Parra, 2004).

Por medio de este proceso de gestión curricular del completamiento de los estudios universitarios, se garantiza la existencia de la investigación formativa, teniendo en cuenta que su ausencia podría afectar el desarrollo de la investigación generativa, pues ambas son (de la Ossa, et al., 2012) parte de la espiral de construcción de conocimiento científico en las instituciones de educación superior.

Por ende, esta investigación, tiene como objetivo fundamental perfeccionar los procesos de titulación en la educación superior, a través de la aplicación de una propuesta praxiológica de integración académico investigativa.

La culminación de estudios o titulación es el proceso mediante el cual los estudiantes universitarios se entrenan, evidenciando los aprendizajes apropiados durante la carrera, de manera teórica y práctica para la resolución de problemas y tareas profesionales, como parte de sus modos de actuación, demostrando sus competencias en el manejo de los métodos específicos de las ciencias que sustentan la profesión, en su interrelación con los métodos propios del ejercicio profesional. Este proceso que es, además, esencialmente evaluativo, deberá concluir con un criterio cuantitativo que disponga el otorgamiento o no del título profesional de que se trate.

Históricamente, el proceso de culminación de estudios en Ecuador, ha sido enfrentado de manera diversa por las múltiples instituciones de Educación Superior, al respecto no existen otras referencias que no sean las recogidas en los indistintos planes de estudio de las carreras. No es hasta que se aprueba la Ley Orgánica de Educación Superior (2010) que se regula de manera holística y coherente el sistema de Educación Superior en Ecuador y se definen sus principios y lógica funcional, para garantizar el derecho a una educación superior de calidad incluyendo el proceso de culminación de estudios o egreso, como también se le ha dado en llamar.

De manera más específica, el Reglamento al Régimen Académico (2013) establece las unidades de organización curricular, en las que se estructura el currículo en la educación superior ecuatoriana: Unidad Básica, Unidad Profesional y Unidad de Titulación. Esta última permite la validación académica de los conocimientos, habilidades y desempeños adquiridos en la carrera para la resolución de problemas profesionales. Su resultado fundamental es el desarrollo de un trabajo investigativo o de intervención de titulación o aprobación de un examen de grado.

Esta nueva estructura curricular comenzará a implementarse en su totalidad a partir del año lectivo 2017- 2018, como resultado del proceso de rediseño curricular llevado a cabo en todo el país, a través de un proceso de gestión del conocimiento compartido 


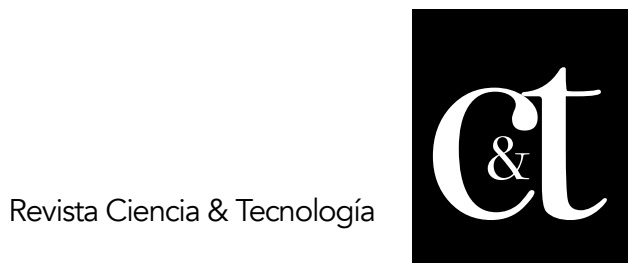

No. 13, 31 de enero de 2017

ISSN impreso: 1390 - 6321

(Matos \& Cruz, 2015). La presente investigación, por tanto, se ubica en el año lectivo 2015- 2016, como período de tránsito en el cual cada Institución de Educación Superior ha asumido sus propias estrategias de implementación de la Unidad Curricular de Titulación, contextualizando a sus condiciones específicas reales, por primera vez, lo establecido en los mencionados documentos legales.

Como consecuencia de lo anterior, se presenta aquí, una perspectiva teórico-práctica de asumir este proceso, a tono con las principales tendencias pedagógicas internacionales, contextualizadas a las condiciones específicas de las IES ecuatorianas, desde la Universidad de Guayaquil (UG).

\section{Metodología}

Siendo así, esta investigación se centra en la metodología de la sistematización de experiencias, como proceso de construcción de nuevo conocimiento científico a partir de la interpretación de los resultados concretos que se fueron alcanzando, por medio de la implementación de acciones académico- investigativas.

Se asume la sistematización de experiencias como un método científico en esencia cualitativo, centrado en la práctica investigativo- profesional sistemática, organizada y encaminada al logro de un objetivo definido, asistida por la observación, como técnica empírica. Lo anterior, constituye un primer estadio, que transita hacia el registro documental de los resultados obtenidos durante un período de tiempo relativamente amplio, a partir de lo cual se definen categorías esenciales del proceso que se estudia, las cuales, a su vez, emergen como un nuevo conocimiento de ciencia (Matos \& Cruz, 2011).

La sistematización lleva implícito un ejercicio de organización, en base a un orden lógico, de los hechos y los conocimientos de la experiencia. Una forma de ordenar que permita llevar a cabo la interpretación crítica de la experiencia.

Esta metodología articuló métodos teóricos, como el análisis- síntesis, el inductivo deductivo y el método hermenéutico-dialéctico durante todo el proceso interpretativo de la investigación. De igual modo, se utilizaron técnicas empíricas como la encuesta y la revisión documental. Para el diseño de la gestión académico investigativa propuesta, se utilizó el método sistémico estructural que permitió desmembrar el proceso en cada una de sus partes constitutivas y revelar sus funciones.

Esta sistematización de experiencias es concreción de una investigación didáctica (Parra, 2004) característica de los procesos universitarios, que tiene como objeto los procesos de formación y por ende, los procesos curriculares, por lo que trasciende la practicidad, para revelarse como un estudio sistemático del hecho educativo que propone nuevas aproximaciones teóricas.

El tipo de investigación utilizada es aplicada, descriptiva, explicativa y propositiva, pues desde la valoración del contexto, objeto de estudio, se determinaron las principales falencias que se evidencian en el proceso de culminación de estudios, así como las causas esenciales que inciden en él, lo que permitió elaborar un sistema de gestión académico- investigativa para potenciar el proceso de titulación.

\section{Resultados}

La UG constituye la mayor universidad estatal del Ecuador, fue creada oficialmente en el año 1897, tiene una oferta académica de tercer nivel de 95 carreras y una matrícula total, por modalidad de estudio, de 69.919 estudiantes. 
En ella, la Escuela de Lenguas y Lingüística, adscrita a la Facultad de Filosofía, Letras y Ciencias de la Educación, tiene como objetivo formar docentes competentes en idioma inglés con un enfoque socio-crítico, sistémico y complejo, para intervenir en la búsqueda de soluciones científicas educativas a problemas de carácter social y humanístico, con compromiso y ética profesional que responda a las exigencias interculturales de la sociedad contemporánea.

En este contexto de formación, la brecha entre los estudiantes que matriculan la carrera, los que egresan (logran vencer los créditos necesarios a través de la aprobación de las asignaturas de la Malla Curricular) y aquellos que finalmente logran titularse ha estado en constante aumento.

A partir de la interpretación de los resultados de una encuesta realizada a una muestra de 80 estudiantes de una población de 140 que egresaron hasta el año lectivo 2014- 2015 y que conformaron la matrícula diurna de la Unidad de Titulación en el año lectivo 2015-2016, es posible afirmar que estos estudiantes posponían su proceso de titulación, por diversas causas relacionadas con el proceso formativo que debe garantizar la institución:

- Insuficiente dominio de la metodología de la investigación, que frenaba sus posibilidades de realizar una tesis de grado pertinente, limitado proceso de orientación personalizada para la culminación de estudios, dado en las imprecisiones funcionales del proceso de tutoría de tesis.

- Exigua formación en el estilo de redacción científica que limitaba sus posibilidades de construir una memoria escrita de investigación.

- Carencias didáctico- metodológicas de la formación en lenguas extranjeras que dificultaban el diseño creativo de propuestas de solución a los problemas de investigación detectados.

De igual modo, en el año lectivo 2015-2016, un grupo de estudiantes que habían completado los créditos entre los dos y cinco años anteriores, sin llegar a titularse por falta de la tesis, lograron presentarlas, por lo que no tuvieron necesidad de matricularse en la Unidad Curricular de Titulación. La Comisión Académica, entonces, procedió a la revisión de dichas tesis para decidir su aceptación o no para el acto de sustentación, a partir de la valoración de su calidad revelada en "la construcción de aprendizajes significativos y la generación de resultados del aprender que satisfagan a los individuos y a los entornos en los cuales estos aplican sus conocimientos" (Ramírez, 2016, p. 269).

Lo anterior, se concreta en el estudio de los siguientes indicadores de análisis:

1. Lógica esencial general (diseño) de la investigación. Redacción científica.

2. Utilización de los métodos, técnicas e instrumentos de investigación teóricos, empíricos y estadísticos.

3. Proceso de interpretación científica de los datos obtenidos mediante la tabulación de los instrumentos empíricos aplicados.

En 98 tesis revisadas por las autoras, como miembros de dicha Comisión, se evidenciaron los siguientes resultados: 
Tabla 1: Resultado de la primera revisión documental de tesis de grado en porcientos de calidad e insuficiencias

\begin{tabular}{|l|l|l|}
\hline \multicolumn{3}{|c|}{ 1ra revisión } \\
Indicadores & $\begin{array}{l}\text { Calidad } \\
40\end{array}$ & $\begin{array}{l}\text { Insuficiencias } \\
60\end{array}$ \\
\hline 1 & 29 & 71 \\
\hline 2 & 42 & 58 \\
\hline 3 & 26 & 74 \\
\hline 4 & & \\
\hline
\end{tabular}

Fuente: Elaboración propia.

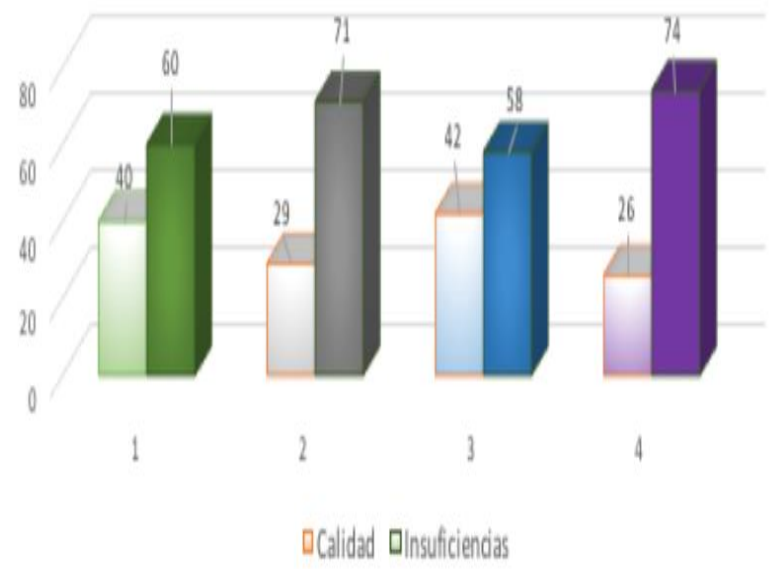

\section{Gráfico 1. Primera revisión}

Fuente: Elaboración propia

Respecto al primer indicador de diseño de la investigación se evidenciaron dificultades en la formulación y coherencia entre las categorías esenciales del proceso investigativo: problema, objetivos, preguntas científicas, métodos, técnicas e instrumentos. Por tanto, la calidad de este indicador fue baja.

En el segundo indicador los estudiantes mostraron insuficiencias respecto a la redacción científica, en especial en cuanto a las citas cortas, largas, parafraseo, crítica y argumentación científica, como manifestación de una inadecuada concepción epistemológica para la construcción del conocimiento científico. Se significa que la calidad de este indicador está por debajo del $30 \%$.

Con relación al tercer indicador, también se mostraron dificultades, aunque se observa un ligero incremento de la calidad, lo que es expresión de una mejor formación metodológica previa, lo que sin embargo, aún no satisface la calidad necesaria para graduarse.

En el caso del cuarto indicador se evidencia una diferencia sustancial en cuanto a la relación calidad e insuficiencia. Es el indicador con resultados más bajos en cuanto a 


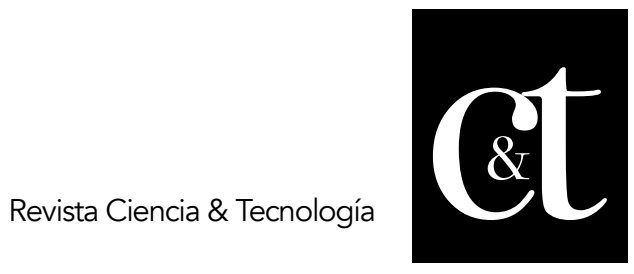

No. 13, 31 de enero de 2017

ISSN impreso: 1390 - 6321

la calidad, lo que implica la necesidad de fortalecer los procesos interpretativos de los datos cuantitativos que se obtienen mediante la aplicación de los instrumentos empíricos.

Por todo lo anteriormente planteado, se hizo necesario diseñar un constructo teóricopráctico que fuera capaz de perfeccionar el proceso de culminación de estudios mediante su implementación inmediata durante la unidad curricular de titulación en el noveno y último semestre de la Carrera.

Se asume que: Se podría considerar que no hay una visión invariable de lo que ha de ser la enseñanza y, en consecuencia, la investigación educativa, pues esta lleva implícita una creencia particular de la sociedad, del ser humano y de la enseñanza, lo que conduce a diversas perspectivas sobre cómo ha de ser abordada (Barba J., González- Calvo \& Barba- Martín, 2014, p. 3).

La culminación de estudios, en el que deberá realizarse un trabajo investigativo en Ciencias de la Educación, deviene por tanto, un proceso complejo, por la propia naturaleza epistémica de la ciencia pedagógica, por lo que se deberá orientar la lógica investigativa de forma coherente y sistémica (Matos \& Cruz, 2011).

Este proceso se estructuró, en lo básico, en torno a lo establecido en el Instructivo de Transición de la Ejecución de la Unidad Curricular de Titulación (s/f), sin embargo, se logró revelar una nueva lógica, coherente con las necesidades más esenciales del proceso de titulación, desde la Escuela de Lenguas y Lingüística.

Se generó así, un proceso de gestión académico investigativa de la Unidad Curricular de Titulación en la que la articulación académico- investigativa logró una estructuración curricular, en la cual cada una de las asignaturas diseñadas para esta unidad de formación se integra a cada uno de los momentos esenciales de la investigación científica que, con carácter formativo, los estudiantes desarrollan, de manera simultánea, en instituciones educativas de enseñanza media-general.

El proceso académico, desarrollado en las aulas, para lograr su integración práctica a la investigación, que finalmente debía conducir a la tesis de grado, en todo momento estuvo centrado en métodos problémicos y participativos de enseñanza, ya que los estudiantes llegan a este estadio de su formación habiendo adquirido los conocimientos y desarrollado las destrezas que se exigen en el currículo de la Carrera, por ende en esta Unidad de Titulación no se incluyen conocimientos totalmente nuevos, sino que se generan los espacios de sistematización problémica para la aplicación de los recursos profesionales, ya apropiados, a favor de la solución creativa de problemas educativos reales.

La articulación académico- investigativa estuvo dinamizada a través de la construcción paulatina de la tesis de grado, a la cual la asignatura de Metodología de la Investigación contribuyó en cuanto a la caracterización del problema y el análisis e interpretación de los resultados empíricos; por su parte, la asignatura de Epistemología contribuyó a los procesos de sistematización teórico metodológica y la asignatura de Cátedra Integradora igualmente al mencionado proceso de sistematización teórico- metodológico para la construcción del marco teórico y, consecuentemente, al diseño de constructos de ciencia, como propuestas transformadoras de la situación disfuncional inicialmente diagnosticada. La asignatura de Narrativa Científica, de igual modo, dinamizó todo el proceso académico- 


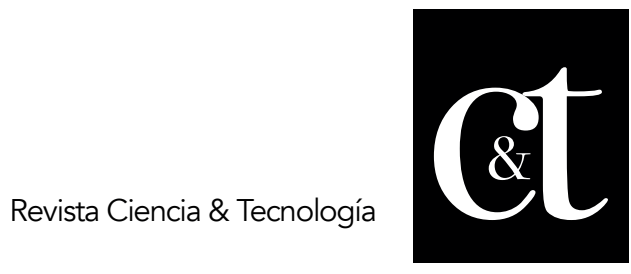

No. 13, 31 de enero de 2017

ISSN impreso: 1390 - 6321

investigativo ya que entrenó a los estudiantes en el estilo científico de redacción de su informe final de tesis, a tono con las tendencias mundiales al respecto.

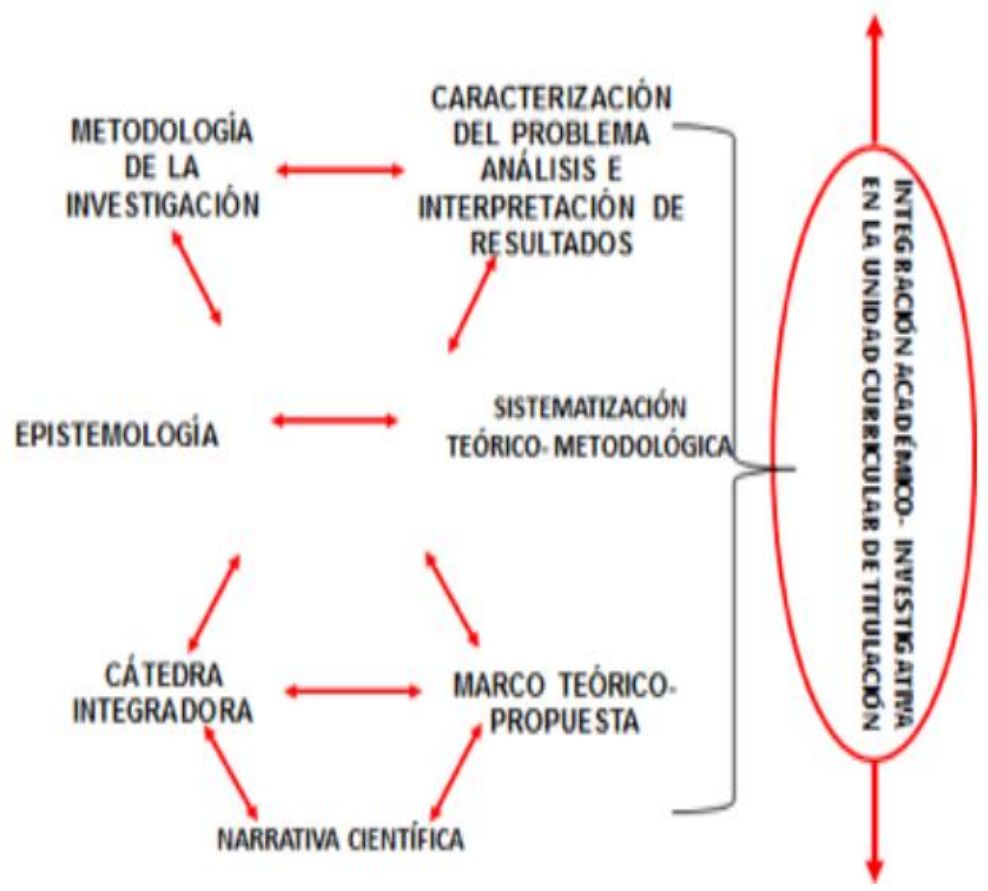

\section{Gráfico 2. Integración académico- investigativa en la Unidad Curricular de Titulación}

Fuente: Elaboración propia

Los resultados obtenidos en este proceso de gestión académico- investigativa son alentadores. La totalidad de los estudiantes matriculados en esta unidad de titulación lograron presentar a tiempo sus diseños de investigación, sintetizados en las fichas de denuncia de tema, con la coherencia y precisión necesarias, para permitir que el $83 \%$ fueran aprobadas sin objeciones y el $17 \%$ fueran aprobadas con sugerencias de perfeccionamiento, por parte de la Comisión Académica.

Se evidenciaron, además, logros de aprendizaje en el proceso de construcción científica de su proyecto de titulación, dados en:

- Utilización crítica de las fuentes bibliográficas consultadas para la justificación del proceso de construcción del conocimiento científico.

- Desarrollo de procesos de análisis y síntesis, para la elaboración de resúmenes de ideas de otros autores que potenciaron su reconstrucción y valoración crítica.

- Establecimiento de comparaciones de conceptos, definiciones, clasificaciones, teorías, dadas por diferentes autores ante un mismo objeto de investigación, que potenció la generalización científica y asentó la concepción de la diversidad científica a partir de los diversos criterios de demarcación de la ciencia establecidas por las comunidades científicas.

- Asunción de una postura epistemológica definida a partir del tipo de paradigma investigativo que defienden como investigadores.

- Reconocimiento de que los conceptos, definiciones, relaciones, teorías y conceptualizaciones que asumen desempeñan un papel esencial en la construcción 
del conocimiento científico y que necesitan ser justificados.

- Construcción colectiva de una propuesta de guía para el análisis epistemológico del texto científico, que sirviera para evaluar el proceso de construcción del conocimiento científico del proyecto de titulación.

- Elaboración de propuestas transformadoras creativas para la solución de problemáticas profesionales, referidas al proceso de enseñanza aprendizaje de lenguas extranjeras.

- Redacción coherente con el estilo científico de informes parciales de resultados de investigación.

- Utilización adecuada de métodos, técnicas e instrumentos teóricos, empíricos y estadísticos.

Se realizó, entonces un análisis comparativo, entre los resultados de la revisión documental realizada a las 98 tesis presentadas ante la Comisión Académica para sustentación, sin haber matriculado la Unidad de Titulación en el año lectivo 20142015, y los resultados de la revisión de la misma cantidad de tesis igualmente presentadas para sustentación, pero luego de la implementación de la gestión académico- investigativa en la Unidad de Titulación en el año 2016. Se utilizaron los mismos indicadores de análisis en ambas revisiones, lográndose los siguientes resultados:

Tabla 2. Resultado de la segunda revisión documental de tesis de grado en porcientos de calidad e insuficiencias

\begin{tabular}{|l|l|l|}
\hline \multicolumn{2}{|l|}{ Segunda revisión } \\
\hline Indicadores & Calidad & Insuficiencias \\
\hline 1 & 82 & 18 \\
\hline 2 & 51 & 49 \\
\hline 3 & 91 & 9 \\
\hline 4 & 76 & 26 \\
\hline
\end{tabular}

Fuente: Elaboración propia

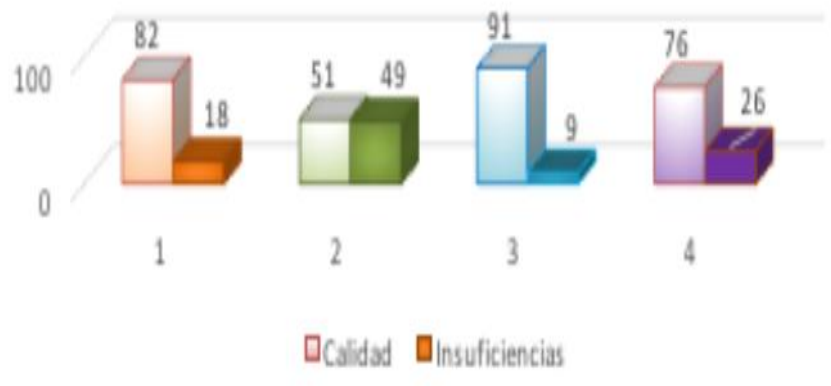

\section{Gráfico 3. Resultado de la segunda revisión documental de tesis de grado}

Fuente: Elaboración propia

Con relación al primer indicador de diseño de la investigación se evidenció un mínimo dificultades en la formulación y coherencia entre las categorías esenciales del proceso 
investigativo. Por tanto, la calidad fue de un $82 \%$, lo que implica un nivel satisfactorio en las tesis.

En el segundo indicador los estudiantes mostraron una relación casi equilibrada entre calidad e insuficiencias respecto a la redacción científica, siendo el indicador con resultados más bajos, como expresión de que aún persisten falencias al respecto, que demandan un tratamiento didáctico más sistematizado, no solo en la Unidad Curricular de Titulación, sino también en la Unidad Básica y la Profesional como parte de la formación que debe potenciar la asignatura de Narrativa Científica.

Con relación al tercer indicador, se reveló un alto índice de calidad en cuanto a la utilización de los métodos, técnicas e instrumentos de investigación teóricos, empíricos y estadísticos, como resultado del proceso de integración académicoinvestigativa, especialmente coordinado por la asignatura de Metodología de la Investigación.

En el caso del cuarto indicador la calidad respecto a los procesos interpretativos de los datos cuantitativos que se obtienen mediante la aplicación de los instrumentos empíricos, tiende a satisfacer las exigencias para este nivel de formación.

En un estudio comparativo, entre los datos obtenidos entre la primera y la segunda revisión documental realizadas, se obtienen los siguientes resultados:

Tabla 3. Resultado de la comparación entre la primera y la segunda revisión documental de tesis de grado en porcientos de calidad e insuficiencias

\begin{tabular}{|l|l|l|l|l|}
\cline { 2 - 5 } \multicolumn{1}{c|}{} & \multicolumn{2}{l|}{ Primera y Segunda revisión } & \multicolumn{2}{l|}{ Primera y Segunda revisión } \\
\hline Indicadores & Calidad & Calidad & Insuficiencias & Insuficiencias \\
\hline 1 & 40 & 82 & 60 & 18 \\
\hline 2 & 29 & 51 & 71 & 49 \\
\hline 3 & 42 & 91 & 58 & 9 \\
\hline 4 & 26 & 76 & 74 & 26 \\
\hline
\end{tabular}

Fuente: Elaboración propia 


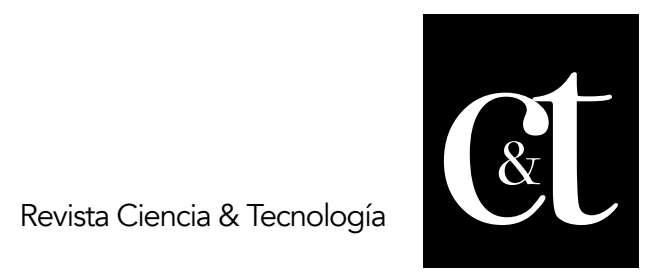

No. 13, 31 de enero de 2017

ISSN impreso: 1390 - 6321

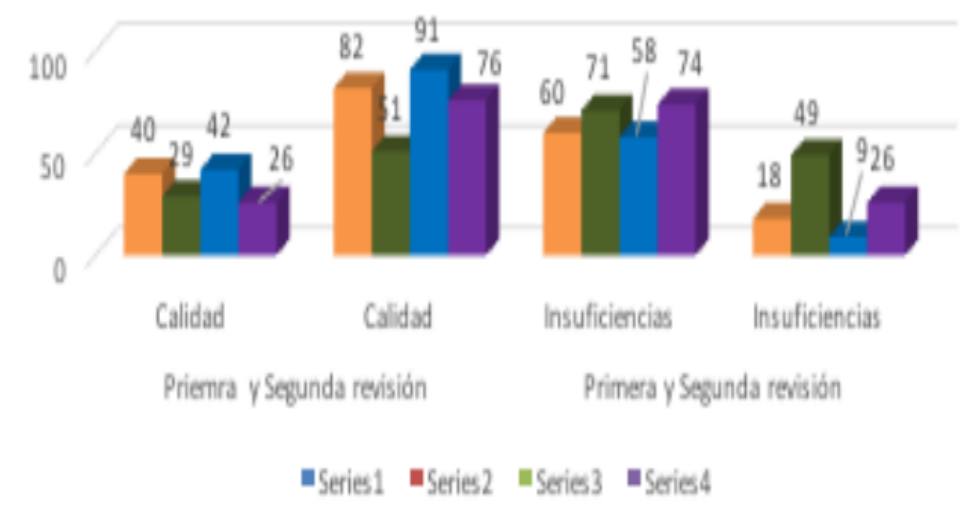

\section{Gráfico 4. Resultado de la comparación entre la primera y la segunda revisión documental de tesis de grado en porcientos de calidad e insuficiencias}

Fuente: Elaboración propia

A partir de la interpretación comparativa de los datos obtenidos entre la primera y la segunda revisión documental realizadas, se evidencian los siguientes resultados por indicadores:

- Aumento de la calidad en un $42 \%$ en la lógica esencial general (diseño) de la investigación, lo que implica una tendencia satisfactoria en este indicador.

- Incremento de la calidad de la redacción científica en un $22 \%$, en especial en cuanto a las citas cortas, largas, parafraseo, crítica y argumentación científica.

En tal sentido, es necesario reconocer que los estudiantes llegan a la Unidad de Titulación con dificultades en sus destrezas de redacción científica que impiden la correcta redacción de la tesis, lo cual implica un proceso de profundización posterior a esta investigación.

- Incremento de un $49 \%$ en la pertinencia y coherencia en la utilización de los métodos, técnicas e instrumentos de investigación teóricos, empíricos y estadísticos. Este indicador logró un incremento sustancial de la calidad, como resultado del proceso de gestión implementado.

- Crecimiento de un $50 \%$ en la calidad del proceso de interpretación científica de los datos obtenidos mediante la tabulación de los instrumentos empíricos aplicados. Ello significa que es este indicador, el que mejores resultados alcanzó a partir de la implementación de la integración académico investigativa. 
Estos resultados, se resumen en el siguiente gráfico:

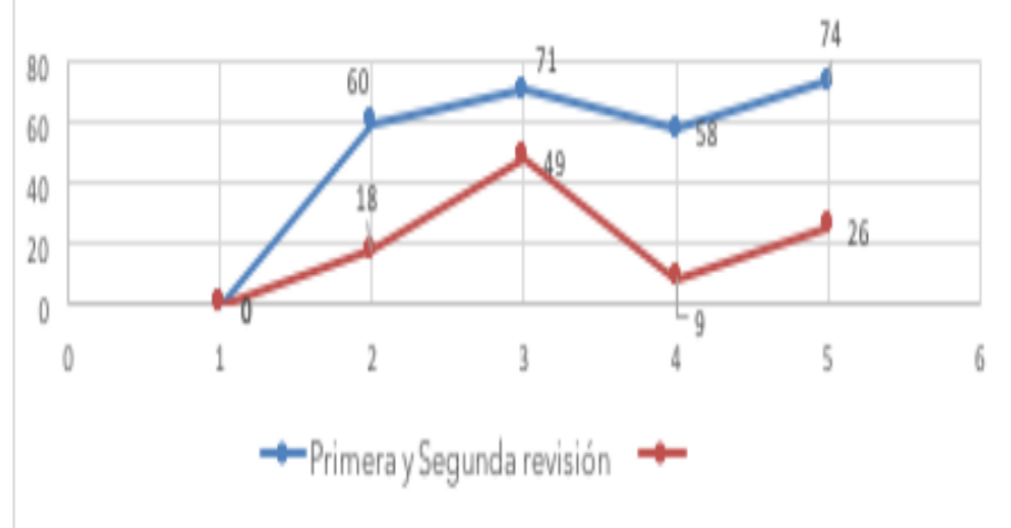

\section{Gráfico 5. Resumen del resultado de la comparación entre la primera y la segunda revisión documental de tesis de grado en porcientos de insuficiencias}

Fuente: Elaboración propia

Se aprecia, entonces, una disminución sustancial de las dificultades, lo que significa una tendencia satisfactoria en la elevación de la calidad del proceso de investigación formativa de los estudiantes, en la Unidad Curricular de Titulación, a partir de la implementación de la integración académico- investigativa propuesta.

\section{Discusión}

Metodología de la investigación- epistemología: una dialéctica curricular- investigativa Las asignaturas de Metodología de la investigación y Epistemología se interrelacionaron, no limitadas cada una a sus propios espacios disciplinares, sino como una integración dialéctica para la construcción de la ciencia, sin fronteras de contenidos, donde la primera aportó esencialmente la construcción de las categorías esenciales del proceso de investigación, los tipos de investigación científica, los métodos, técnicas e instrumentos, como vías de construcción del conocimiento, y la segunda, la lógica de la investigación y los procesos de argumentación científica a través de las formas de conocer, la dinámica del conocimiento científico y su proceso de justificación, lo que implicó que necesariamente cada una de estas asignaturas retomara a la otra para hacer este proceso viable, concreto, factible, creativo, actualizado y pertinente.

A pesar de que el objetivo de esta unidad curricular de titulación es la sistematización teórico- práctica de los contenidos ya apropiados durante toda la carrera y, por ende, no deben introducirse aspectos totalmente nuevos, en este momento de tránsito hacia la asunción total del nuevo rediseño curricular, los estudiantes no habían recibido previamente en el currículo la asignatura de Epistemología, por lo que hubo necesidad de introducir algunos conceptos, definiciones, categorías, conceptualizaciones y relaciones esenciales, que como base estructural teórica, funcionaran para la praxis epistemológica, interrelacionando así, la teoría con la 


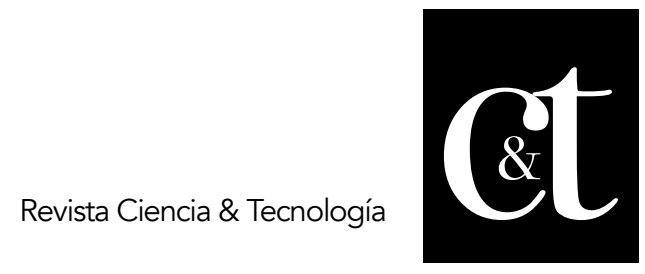

No. 13, 31 de enero de 2017

ISSN impreso: 1390 - 6321

actividad cognoscitiva del proceso de construcción del proyecto de investigación. Se potenciaron, por tanto, los procesos inductivos-deductivos, de análisis y síntesis, de comparación y de generalización científica que se concretaron en los tránsitos entre los datos teóricos y empíricos, información y nuevo conocimiento científico develados en la investigación educativa realizada, lo que a su vez se fue revelando en el texto científico construido.

Es por ello que la Epistemología de la investigación tuvo como objeto de estudio el proceso de construcción del conocimiento científico, haciendo énfasis en tres interrogantes principales, que como hilos conductores problémicos de debate científico fueron dinamizando tópicos generativos de contenidos: ¿qué es el conocimiento científico?, ¿cómo se produce el conocimiento científico? y cómo determinamos la "verdad en ciencias"? Por tanto, se elaboraron hilos conductores para la construcción de esa base estructural teórica que sirviera para el desarrollo del debate en el trabajo colaborativo de los estudiantes y que, a su vez, fueran contribuyendo a la derivación de cada núcleo de contenido, potenciando su inserción con el resto de las asignaturas de la Unidad de Titulación, a través de las siguientes interrogantes epistemológicas que marcaron la esencialidad del contenido a compartir, en su vinculación con los temas particulares de investigación de cada estudiante:

1. ¿Cuál es la relación entre ciencia-investigación epistemología?

2. ¿Qué es ciencia?

3. ¿Qué es el conocimiento científico?

4. ¿Cómo se produce el conocimiento científico?

5. ¿Cómo se justifican los enunciados científicos?

6. ¿Cuáles son los diversos criterios de demarcación de la ciencia?

7. ¿De dónde se deriva su validez?

8. ¿Dónde se ubica la Pedagogía en el espectro epistemológico?

Estos hilos conductores conllevaron a la propuesta de los siguientes núcleos de debate epistemológico que potenciaron la construcción de la base estructural teórica que se necesitaba para el proceso de construcción científica:

1. Relación ciencia-investigación- epistemología.

2. Diversos tipos de conocimiento. El conocimiento científico, su construcción.

3. Diversos criterios de demarcación de la ciencia. El papel de las comunidades científicas y la historia en la construcción del conocimiento científico.

4. La naturaleza epistémica de las investigaciones de carácter pedagógico: su carácter inter y transdisciplinario.

Durante este proceso formativo se fue construyendo a su vez, por los propios estudiantes, una guía de análisis epistemológico para el texto científico, en la misma medida en que se iban sintetizando ideas científicas esenciales valoradas, lo que permitió ir evaluando sus logros de aprendizaje en la construcción de su proyecto de titulación en un proceso de auto-co-hetero- evaluación. Esta guía se sintetizó en los siguientes indicadores de análisis:

- Saber argumentar el contenido conceptual metodológico de su ciencia particular en relación dialéctica con la naturaleza del problema científico investigado y el resto de las categorías del diseño de investigación, enfatizando en: 


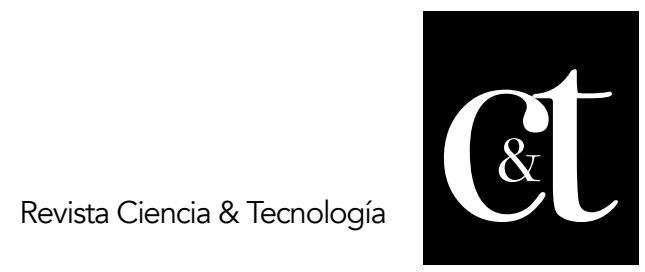

No. 13, 31 de enero de 2017

ISSN impreso: 1390 - 6321

- La elaboración coherente de las categorías del diseño de investigación: relación entre problema de la investigación, objetivos, métodos, técnicas, instrumentos, y aporte científico propuesto.

- La justificación del tema de investigación desde la selección de los criterios de análisis de pertinencia, actualidad y novedad.

- La selección adecuada de paradigmas, enfoques, teorías, métodos, estrategias, indicadores y criterios de análisis históricos y contextuales que singularizan ese objeto investigado desde una posición epistemológica definida y coherente.

- Los análisis interpretativos críticos, que, como síntesis epistémicas y metodológicas de la valoración teórica sistematizada, dinamizan toda la investigación.

- La selección, explicación y utilización adecuada del tipo de investigación a desarrollar, los métodos y técnicas teóricos, empíricos y estadísticos utilizados acorde al paradigma de investigación asumido.

- La valoración e interpretación de los principales resultados científicos alcanzados en la tabulación de los datos obtenidos de la aplicación de los instrumentos científicos.

- La construcción argumentada de una propuesta creativa y pertinente en su relación dialéctica con el problema científico revelado y los objetivos trazados, sustentada en los "criterios de validez de la ciencia", según el paradigma asumido.

- Saber encontrar y sustentar similitudes y diferencias conceptuales y metodológicas entre las Ciencias de la Educación, la Pedagogía, la Didáctica y las otras ciencias que fundamentan científicamente el objeto investigado a partir de la naturaleza epistémica de las investigaciones educativas.

- Saber hacer conclusiones y recomendaciones de ciencia que singularicen las interpretaciones realizadas que expresan la identidad científica del investigador.

- Saber referenciar la bibliografía utilizada, como expresión de los criterios de actualidad y relevancia científica acorde al objeto investigado.

Esta propuesta de guía de análisis epistemológico sirvió para evaluar la calidad del proyecto investigativo que se iba construyendo. El carácter interdisciplinario desarrollado con el resto de las asignaturas: Narrativa científica, Metodología de la investigación y Cátedra Integradora, que colindaron y se correlacionaron dialécticamente, permitió a su vez, la observación, la comprensión, la explicación y la interpretación científica del campo profesional y su contexto de aplicación en escenarios educativos concretos a partir de la elaboración del proyecto de investigación, que se constituyó en el eje articulador de la gestión académica investigativa.

Por tanto, esta asignatura de Epistemología de la investigación se caracterizó por ser de naturaleza esencialmente praxiológica, donde se imbricaba la práctica investigativa en relación con la base estructural teórica que se iba construyendo, lo que se fue revelando en las diversas fundamentaciones teóricas de los proyectos de tesis.

Conjuntamente con esta dinámica, la asignatura de Metodología de la Investigación enfatizó en la contextualización de la situación conflicto, sus causas, el planteamiento del problema de investigación, los objetivos, las preguntas de investigación, la justificación del mismo, los tipos de investigación, métodos, técnicas e instrumentos científicos utilizados, así como en el proceso de tabulación de los resultados y el análisis e interpretación de los datos empíricos obtenidos. 
La naturaleza de esta asignatura fue esencialmente práctica y participativa, sistemática e interdisciplinaria, pues retomó los contenidos ya apropiados por los estudiantes en el currículo y fue aplicando los nuevos conocimientos epistemológicos que iban construyendo.

Los talleres realizados permitieron ir revelando los avances en la construcción del proyecto de titulación.

Esta asignatura, en unión con el resto, propuso a los estudiantes los cuatros grandes campos de desempeño comunicativo (Expresión Oral, Expresión Escrita, Lectura y Audición) que son coherentes con los que plantea el Marco Común de Referencia Europeo (2012) y el Currículo Nacional de Inglés (2012) y sus Especificaciones Curriculares (2012), lo que posibilitó orientar los proyectos de tesis a esos cuatros núcleos esenciales de investigación científica, que estaban en correspondencia con los análisis de pertinencia realizados por la carrera y con las líneas de investigación asumidas.

A partir de estos núcleos esenciales de investigación, los estudiantes seleccionaron a cuál de ellos se acogían, en dependencia de los resultados del diagnóstico realizado en los campos de actuación profesional en que realizaban su proyecto de tesis, y a partir de un proceso de indagación científica precisaban la particularidad que los identificaba. Esta estructura de organización de los proyectos de tesis en estos cuatros núcleos esenciales de investigación permitió a su vez, la selección de los tutores acordes a las potencialidades científicas de cada uno de ellos en una de estas temáticas.

Este proceso potencia la pirámide investigativa, donde los docentes-tutores podrán producir investigación generativa, incorporando a su proceso investigativo los resultados de la investigación formativa realizada por sus tutorados en estos proyectos de tesis.

Cátedra Integradora: una perspectiva holístico-curricular del desempeño profesional La asignatura de Cátedra Integradora, ofreció una visión holística de los contenidos esenciales desarrollados durante todo el proceso formativo con fines investigativos.

Se centra en una perspectiva transdiciplinar donde se integran todos los contenidos necesarios alrededor de la solución de un problema práctico y real del desempeño profesional. Se logra aquí articular conocimientos, destrezas y valores en torno al ejercicio de la competencia investigativa, que en su momento fueron tratados de manera mayormente fragmentada en diversas asignaturas de la especialidad.

Se asume aquí la complementariedad, que como principio: "subraya la incapacidad humana de agotar la realidad con una sola perspectiva, punto de vista, enfoque, óptica o abordaje, es decir, con un solo intento de captarla. La descripción más rica de cualquier entidad (...) se lograría al integrar en un todo coherente y lógico los aportes de diferentes personas, filosofías, métodos y disciplinas" (Martínez Miguélez, 1997, p. 98).

De este modo, el estudiante es capaz de asumir el enfoque complejo (Morín, 1999) como la única alternativa de pensamiento y acción viable para dar respuestas coherentes e integradoras a los problemas de su práctica profesional educativa. 


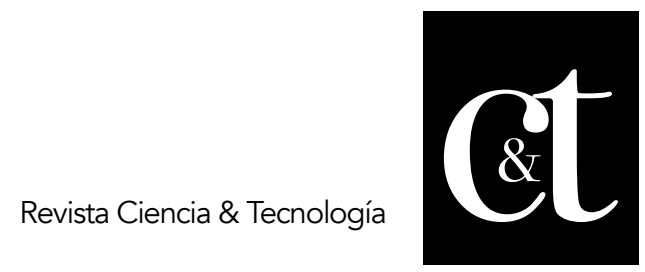

No. 13, 31 de enero de 2017

ISSN impreso: 1390 - 6321

El estudiante, entonces, sistematiza aspectos teóricos y metodológicos relacionados con la filosofía, la lingüística, la pedagogía, la didáctica de las lenguas extranjeras, la psicología, la sociología, la literatura, la historia u otras ciencias necesarias, logrando una actitud consciente, que emana de la práctica investigativa en contextos reales, ante la inexistencia de fronteras inter-ciencias. De este modo, al propiciarse un amplio enfoque sobre la urgencia teórico- fáctica de enfrentar la investigación pedagógica contemporánea mediante complejos procesos holísticos, los estudiantesinvestigadores fueron capaces de reconocer la necesidad de la búsqueda de áreas de convergencias culturales y científicas, lo que les llevó a desarrollar la indagación, la argumentación, la crítica y la creatividad en la propuesta de soluciones, desde una cosmovisión inter-científica basada en fundamentos epistemológicos que fueron reflejados en los marcos teóricos de sus tesis.

Esta asignatura se organizó en torno a la investigación en dos grandes núcleos de contenidos que se identifican con las destrezas receptivas (lectura y audición) y las destrezas productivas (escritura y expresión oral). Estos dos grandes grupos, entonces, en sus subdivisiones internas, coinciden con los cuatro grandes campos de organización investigativa, propuestos a los estudiantes en la asignatura de metodología de la investigación, lográndose así, una interdisciplinariedad funcional entre ambas asignaturas.

En este proceso ambas asignaturas se aglutinaron alrededor de los temas de investigación propuestos por los estudiantes, dotándolos de las herramientas necesarias para intervenir en la práctica educativa.

Esta cátedra, además, se interrelaciona con la asignatura de Epistemología, pues facilitó al futuro profesional la comprensión integradora de la naturaleza científica compleja de su desempeño docente, contribuyendo a la consecución de su perfil de egreso, a través del saber, el hacer, el ser y el convivir como docente comprometido con el mejoramiento social, mediante:

- Manejo de fundamentos teóricos, metodologías y estrategias de enseñanza que desarrollen un aprendizaje de lenguas extranjeras reflexivo, por descubrimiento, participativo y en contextos de aplicación de las destrezas comunicativas.

- Solución creativa de problemas emergentes de la praxis profesional, relativos a la competencia comunicativa, sustentando epistemológica y metodológicamente.

Es importante reconocer que este proceso de gestión académico- investigativa, dentro de la Cátedra Integradora, implica un reto para los docentes que la faciliten, pues como condición indispensable, es necesario poseer una profunda formación en las esencialidades y actual desarrollo de la diversidad de ciencias que sustentan la profesión, así como una sentida formación didáctica que permita su integración funcional desde el salón de clases, en función de la diversidad de marcos teóricos y propuestas de investigación a construir.

El docente, entonces, debe ser capaz de lograr un equilibrio entre lo individual y lo grupal, entre su propia formación previa y los desafíos de esta nueva propuesta, para poder satisfacer, de manera dinámica e integradora las necesidades de los estudiantes de aprender a hacer investigación educativa, con sólida formación epistemológica holística. 


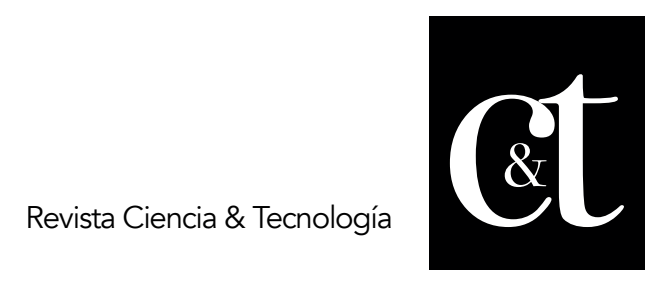

No. 13, 31 de enero de 2017

ISSN impreso: 1390 - 6321

En tal sentido, se reconoce que, en algunos casos, existieron dificultades en cuanto al desempeño docente que frenaron el potencial desarrollo de los estudiantes, pues ha resultado difícil asumir los retos de enfrentar un nuevo proceso integrador $y$, por ende, audaz, que deberá comenzar a marcar los caminos para el perfeccionamiento integrador de la formación investigativa, no solo en la unidad de titulación, sino también en la formación básica y la profesional.

\section{¿Redacción o narrativa? Una construcción textual argumentativa para la} comunicación de los resultados de la investigación

En el Reglamento al Régimen Académico (2013) se hace referencia a la necesidad de desarrollar habilidades comunicativas para la elaboración de narrativas académicas y científicas. Sin embargo, este término "narrativa" se ha estado identificando indistintamente con el de tesis, texto científico, memoria de investigación u otros similares.

Es interés de este artículo, precisar que debe entenderse por narrativa el producto de la producción científica, académica y profesional sistemática durante un amplio período que logra caracterizar la evolución y consecuentes transformaciones en la madurez epistemológica y praxiológica de quien la produce.

Por ende, puede estar caracterizando a individuos, grupos de investigadores docentes, profesionales- o pueden llegar a caracterizar las tendencias en la producción científica institucional, empresarial u otras que, como grandes grupos humanos, han ido generando cíclicamente su propia sabiduría (Matos \& Cruz, 2015).

Por tanto, una tesis de grado o postgrado no es una narrativa en sí misma, sino que sique constituyendo un informe, reporte o memoria de investigación que pasa a formar parte de la narrativa de su autor o de los sujetos involucrados en su producción y, a la vez, de la institución a las que estos pertenecen.

Una narrativa, entonces, se genera mediante el proceso de construcción socializada del conocimiento que, de manera holística, refleja la historia evolutiva de su gestión y que va generando una cultura de la producción científica.

Siendo así, los estudiantes fueron adiestrados en la construcción de textos, por medio de la justificación, la crítica científica, la valoración, en su interrelación con los procesos hermenéuticos de comprensión, explicación e interpretación, lo que obviamente dista de una narración y se identifica plenamente con un texto argumentativo.

En esta asignatura de Narrativa Científica se desplegó un entrenamiento en redacción científica por medio de la escritura por procesos, privilegiando el estilo científico. Por tanto, respecto al léxico y la estructura gramatical, se ejercitó la redacción para lograr que la expresión se adaptase adecuadamente a la precisión y exactitud del pensamiento científico.

Los docentes enfatizaron la necesidad de utilizar palabras unívocas y especializadas, así como expresiones abstractas que sirven, por ejemplo, para resumir lo dicho, para expresar explícitamente las relaciones de existencia, posibilidad, necesidad, causalidad, finalidad, etc., que se establecían al interior de los constructos trabajados. 


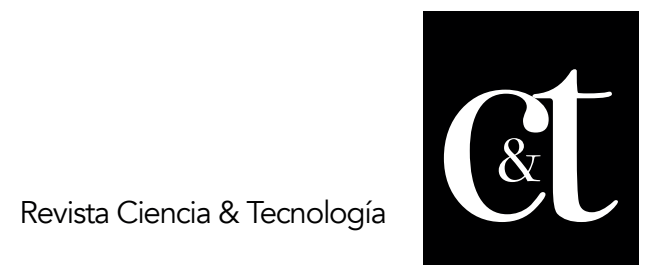

No. 13, 31 de enero de 2017

ISSN impreso: 1390 - 6321

Asimismo, se potenció la construcción estilística de la sintaxis textual, cuidándose el uso correcto de oraciones bimembres, de voz pasiva y oraciones subordinadas hasta de cuarto grado, utilizando preferentemente ciertas conjunciones interrelacionados.

Es pertinente destacar, que los estudiantes sistematizaron en la práctica de la redacción de sus tesis, desde el contexto académico- investigativo, las normas de la American Psychological Association (APA) (2010) sexta edición, respondiendo a lo establecido en la estructura de tesis de titulación de la Facultad.

De este modo, se sentaron las bases para que los estudiantes puedan incorporarse al tan necesario proceso de comunicación epistémica de los resultados de sus investigaciones, dando a conocer así a la comunidad académica internacional, los aspectos más significativos revelados en sus tesis de grado. Se compartió, además, para esto, orientaciones respecto a las publicaciones indexadas en bases de datos de prestigio internacional, que pudieran aceptar artículos científicos de naturaleza exploratoria, descriptiva y propositiva, coherentes con el tipo de investigación realizada en estas tesis de titulación, de modo que los estudiantes se motiven a comenzar a transitar, con ayuda de sus tutores, en el complejo camino de la publicación internacional en revistas de prestigio.

\section{Conclusiones}

En sentido general, en todas las asignaturas se desplegaron los métodos de proyecto, por tareas e investigativo, los profesores se constituyeron en acompañantes de este proceso, estableciendo un proceso evaluativo sistemático e interdisciplinario que evidenció los logros en la construcción científica de los proyectos de titulación, además, la evaluación no utilizó la aplicación de los exámenes tradicionales, sino una evaluación final integradora de todas las asignaturas que consistió en la sustentación de la primera versión de su proyecto investigativo.

Finalmente, es necesario afirmar que la presente investigación praxiológica ha sido capaz de revelar una lógica esencial para la gestión del proceso de culminación de estudios en educación superior, a partir de la integración de la formación académica e investigación formativa. Por ende, ya se ha comenzado a transitar la brecha en las investigaciones de Pedagogía de la Educación Superior respecto a este estadio final de la formación en el que muchos estudiantes tradicionalmente abandonan o posponen las acciones finales para la obtención de los títulos académicos que los acreditan como profesionales, afectando así, no solo los índices de acreditación de la calidad de las instituciones, sino también y muy especialmente, su propia inserción laboral y el crecimiento socio- profesional de sus comunidades.

La modelación sistémica realizada al proceso de gestión académico- investigativa de la Unidad Curricular de Titulación implica una respuesta teórico-metodológica que argumenta una vía para superar las falencias en el proceso de implementación curricular de la culminación de estudios lo que ha logrado potenciar la respuesta universitaria a las demandas sociales de profesionales graduados de esta carrera.

Estos resultados de investigación son factibles de ser generalizados a una diversidad de carreras universitarias, teniendo como condición básica el profundo dominio de la lógica esencial de la profesión y del plan de estudios de la carrera de la que se trate, de modo que sea posible contextualizar esta propuesta. 


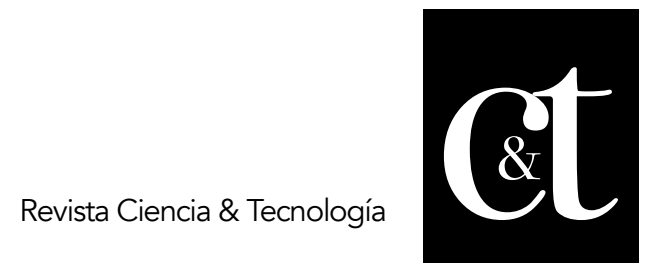

No. 13, 31 de enero de 2017

ISSN impreso: 1390 - 6321

\section{Limitaciones del estudio y proyecciones}

A pesar de los logros declarados en el acápite de Resultados, hasta el momento en que se redacta este artículo existe un $18 \%$ de la totalidad de estudiantes matriculados a esta Unidad de Titulación que aún no logran entregar la versión final de sus tesis, por dificultades en la sistematicidad de las tutorías, que debieron haber recibido, posteriores a las horas clases planificadas, como parte de las asignaturas ya descritas. Se hace necesario, entonces, asumir un nuevo enfoque en cuanto a las tutorías de tesis, que vaya más allá de la tradicional revisión del informe escrito que se acostumbra realizar.

\section{El coaching en titulación}

Se propone, entonces, entrenar a los docentes en los modernos procesos de coaching educativo, de modo que su labor trascienda hacia un acompañamiento constante y personalizado del estudiante, donde se le brinden no solo las orientaciones referidas a la investigación en sí, sino que también se le motive a perseverar en su proceso de culminación de estudios, más allá de las dificultades que pudieran aparecer. Asumir la metodología del coaching educativo en este ámbito implica desarrollar entre docentes y estudiantes un compromiso profesional y afectivo que ha de redundar en la elevación de la calidad del proceso: "porque se entiende que la aplicación del coaching transporta a las personas, desde donde se encuentran hoy, hacia donde les gustaría estar mañana, siendo el coach el facilitador de este viaje" (Bayón, F.; Cubeiro, J.C.; Romo, M.; Sainz, J.A., 2006, p.7).

Se propone asumir esta metodología, ya que considerando lo planteado por Sánchez Mirón \& Boronát Mundina (2014) "se basa en el establecimiento de metas personales y en la ejecución de una serie de acciones planificadas que permiten al sujeto poner en práctica determinadas formas de ser y de actuar." (p.1).

Se hace necesario, además, garantizar la actualidad científica de los docentes en relación con el enfoque complejo desde las ciencias pedagógicas y potenciar su flexibilidad para integrar en un todo único, las partes de un proceso que ya no puede seguir estando fragmentado.

De igual modo, los docentes deberán integrarse a los procesos institucionales de investigación generativa, pues de lo contrario se estaría tratando en las aulas un proceso que les es ajeno: solo quien se desempeña en lo que promulga, es capaz de hacerlo con profundidad, sin temor a los riesgos de la incertidumbre.

\section{Referencias bibliográficas}

Barba J., González- Calvo, A. \& Barba- Martín, M. (2014). Que la fuerza esté contigo: desvelar el lado oscuro de la investigación en educación. Revista Internacional de Investigación en Educación, 7 (14), 3. Recuperado de http://www.redalyc.org/pdf/2810/281032883010.pdf

Bayón, F.; Cubeiro, J.C.; Romo, M.; Sainz, J.A. (2006). Coaching realmente. Madrid: Pearson Prentice Hall. p.7.

Blum, L. D. (2010). The All-But-the-Dissertation Student and the Psychology of the Doctoral Dissertation, Journal of College Student Psychotherapy, 24: 2, $74-85$. DOI: $10.1080 / 87568220903558554$.

Bridgmon, K. D. (2007). All but dissertation stress among counseling and clinical 


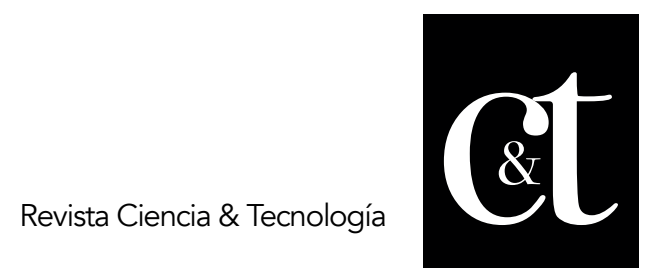

No. 13, 31 de enero de 2017

ISSN impreso: 1390 - 6321

psychology students. Dissertation Abstracts International, 68(3), no. AAT3257723

Consejo de Educación Superior. (2013). Reglamento al Régimen Académico. Quito, Ecuador. Recuperado de:

http://www.utpl.edu.ec/sites/default/files/documentos/reglamento-de-

regimenacademico-2013.pdf

De la Ossa, V. J., Pérez, C. A., Patiño, P., Montes, V. (2012). Editorial. La investigación formativa y la formación para la investigación en el pregrado. Revista Médica Herediana, versión On-line ISSN 1729-214X. Recuperado de: http://www.scielo.org.pe/pdf/rmh/v20n3/v20n3e1.pdf

Facultad de Filosofía, Letras y Ciencias de la Educación. (s/f). Instructivo de Transición de la Ejecución de la Unidad Curricular de Titulación. Universidad de Guayaquil, Ecuador. Recuperado de: http://filosofia.edu.ec/wpcontent/uploads/2015/07/ INSTRUCTIVO1.pdf

Instituto de Estudios sobre Desarrollo y Cooperación Internacional, Universidad del País Vasco (2004). La sistematización, una nueva mirada a nuestras prácticas. Recuperado de: file/d/OB1VcrqAOJd_zOHB6eEtxYTFIU00/view https://drive.google.com/

James L. (2010) ABD: All But Dissertation. Statistics Solutions. Recuperado de: http://www. statisticssolutions.com/wp-content/uploads/kalinspdf/ singles/abd-all-but-dissertation.pdf

Jiménez P., Díaz R, \& Catalá C. (2011). La preparación para la culminación de estudios de Contabilidad y Finanzas de la SUM, Cabaiguán, Cuba. Revista Iberoamericana de Educación (RIES), 2, 5, 126-140. Recuperado de: http://ries.universia.net/index. php/ries/article/view/150

Martínez, M. (1997). El paradigma emergente: hacia una nueva teoría de la racionalidad científica. e.2. México: Trillas; p.98 (1 ${ }^{a}$ edic. Barcelona: Gedisa, 1993).

Matos \& Cruz. (2011). La práctica investigativa, una experiencia en la formación doctoral en Ciencia Pedagógica. Ediciones UO, Cuba. Recuperado de: https://es.scribd.com/doc/69007674/Libro-Practicainvestigativa-Eneida-Lorna2011

Matos \& Cruz (2015). Construcción epistémico participativa de la armonización curricular en Ecuador: su ejemplificación en la Universidad de Guayaquil. Did@scalia: Didáctica y Educación, 6, 3, 1-20. Recuperado de:

http://runachayecuador.com/refcale/index.php/didascalia/article/view/361/316

McDermott, B. J. (2002). The utility of perceived stress, locus of control, and Type A behavior pattern as predictors of doctoral degree completion in a non-traditional EdD program. Dissertation Abstracts International, 63(6), no. AAT 3055934.

Ministerio de Educación del Ecuador. (2012). Currículo Nacional de Inglés. Quito, Ecuador. Recuperado de: http://educacion.gob.ec/wpcontent/uploads/downloads/2014/09/01-NationalCurriculum-Guidelines-EFL-Agosto-2014.pdf

Ministerio de Educación del Ecuador. (2012). Especificaciones al Currículo Nacional de Inglés. Quito, Ecuador

http://educacion.gob.ec/wp- 


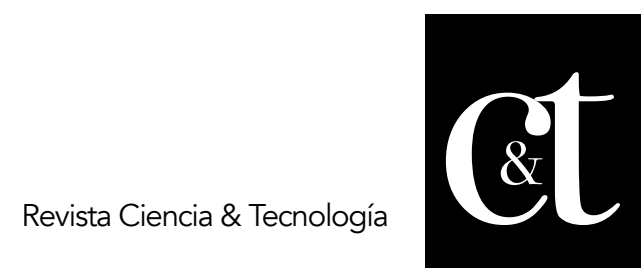

No. 13, 31 de enero de 2017

ISSN impreso: 1390 - 6321

content/uploads/downloads/2013/09/Curriculum_Specifications_EFL-SPECS_Level-A1.pdf

Morín, E. (1999). Los siete saberes necesarios para la educación del futuro. UNESCO. París, Francia.

Mujtaba, B. G. \& Mujtaba, M. G. (2007). Challenges and Joys of Earning a Doctorate Degree: Overcoming the "ABD" Phenomenon. Academy of Business Disciplines Proceedings; November 1-3; Fort Myers Beach, Florida. Recuperado de:

http://citeseerx.ist.psu.edu/viewdoc/download?doi=10.1.1.490.955\&rep=rep1\& type $=p d f$

Parra C. (2004) Apuntes sobre la investigación formativa. Educación y educadores, 7: 57-77. Recuperado de: http:/educacionyeducadores. unisabana.edu.co/index.php/eye/article/view/549

Publication Manual of the American Psychological Association. Washington, EUA. Recuperado de:http://www.hse.ru/data/2013/12/06/1336424472/APA\%20Manual\%206th \%20Edition\%20\% 281\%29.pdf

Ramírez R. (2016) Universidad urgente para una sociedad emancipada. SENESCYTIESALC, Quito, Ecuador. Recuperado de: http://catedras.ciespal. org/unesco/wpcontent/uploads/sites/7/2016/04/ UniversidadUrgenteRR.pdf

Restrepo Gómez B. (2003) Investigación formativa e investigación productiva de conocimiento en la universidad. Nómadas, núm. 18, mayo, 2003, pp. 195-202. Recuperado de: http://www.redalyc.org/articulo.oa?id=105117890019

Restrepo Gómez B. (2016) Conceptos y Aplicaciones de la Investigación Formativa y Criterios para Evaluar la Investigación científica en sentido estricto. Recuperado de: http://www.cna.gov.co/1741/articles-186502_doc_academico5.pdf

Sánchez Mirón, B. y Boronat Mundina, J. (2014). Coaching Educativo: Modelo para el desarrollo de competencias intra e interpersonales. Educación XX1, 17 (1), 221-242. doi: DOI: 10.5944/educxx1.17.1.1072. p. 1. 